AperTO - Archivio Istituzionale Open Access dell'Università di Torino

\title{
Position and flux stabilization of X-ray beams produced by double-crystal monochromators for EXAFS scans at the titaniumK-edge
}

\section{This is the author's manuscript}

Original Citation:

Availability:

This version is available http://hdl.handle.net/2318/152488

since 2016-10-08T16:09:15Z

Published version:

DOI: $10.1107 / S 1600577513034747$

Terms of use:

Open Access

Anyone can freely access the full text of works made available as "Open Access". Works made available under a Creative Commons license can be used according to the terms and conditions of said license. Use of all other works requires consent of the right holder (author or publisher) if not exempted from copyright protection by the applicable law. 


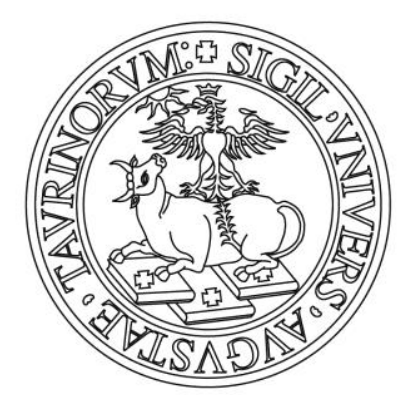

\title{
UNIVERSITÀ DEGLI STUDI DI TORINO
}

This is an author version of the contribution published on:

Questa è la versione dell'autore dell'opera:

\section{Position and flux stabilization of X-ray beams produced by double-crystal monochromators for EXAFS scans at the titanium $K$-edge}

\section{Roelof van Silfhout, Anton Kachatkou, Elena Groppo, Carlo Lamberti and Wim Bras}

\author{
J. Synchrotron Rad. (2014). 21, 401-408 \\ doi: $10.1107 / \mathrm{S} 1600577513034747$ \\ The definitive version is available at: \\ La versione definitiva è disponibile alla URL:
}

http://scripts.iucr.org/cgi-bin/paper?S1600577513034747 


\title{
Position and flux stabilization of x-ray beams produced by double crystal monochromators for EXAFS scans at Ti-edge
}

\author{
Roelof van Silfhout $^{a_{\star}}$, Anton Kachatkou ${ }^{a}$, Elena Groppo ${ }^{b}$, Carlo Lamberti ${ }^{b}$ and Wim Bras $^{c}$ \\ ${ }^{a}$ School of Electrical and Electronic Engineering, The University of Manchester, Sackville Street Building, \\ Manchester, M13 9PL, England, 'bepartment of Chemistry, NIS Center of Excellence, and INSTM unit, \\ University of Turin, Via Giuria 7, Turin, I-10125, Italy, and ${ }^{\mathrm{c}} 3$ Netherlands Organisation for Scientific \\ Research (NWO), Dubble, ESRF, 6 Rue Jules Horowitz, Grenoble, 38043, France \\ Correspondence email: r.vansilfhout@manchester.ac.uk
}

Keywords: EXAFS; beam position control; beam intensity stabilisation; monochromator; Ti Kedge

\section{Synopsis}

Dual loop active feedback stabilisation of the position and monochromatic flux of the beam at the output of the double crystal monochromator is described. The efficacy of the proposed stabilisation system is demonstrated in EXAFS spectroscopy measurements on inhomogeneous diluted Ti containing samples.

\footnotetext{
Abstract

We report on the simultaneous and active feedback stabilisation of $\mathrm{x}$-ray beam position and monochromatic beam flux during EXAFS scans at the Titanium K-edge as produced by a double crystal monochromator beam line. The feedback is generated using two independent feedback loops using separate beam flux and position measurements. The flux is stabilized using a fast extremum-searching algorithm that is insensitive to changes in the synchrotron ring current and energy dependent monochromator output. Corrections of beam height are made using an innovative transmissive beam position monitor instrument. We demonstrate the efficacy of the feedback stabilisation method by comparing the measurements of EXAFS spectra on inhomogeneous diluted Ti containing samples with and without feedback applied.
}

\section{Introduction}


Non-dispersive double crystal monochromators (DCM) are omnipresent at synchrotron radiation beam lines around the globe due to their ease of use, particularly for experiments that require $\mathrm{x}$ ray beams of varying energy. These devices can be made to sagittally focus the divergent monochromatised radiation by bending one of the two crystals (Pascarelli et al., 1996). The most common variant uses two independent, highly perfect crystals that are carefully aligned such that their crystal planes match up for the highest emergent monochromatic flux in a pseudo channel-cut setup.

A major concern with these designs is to keep the two crystals aligned at all times because the angular acceptance range (the misalignment range in angles for which a monochromatic output beam can be measured) is very narrow (approx. $70 \mu \mathrm{rad}$ at $5 \mathrm{keV}$ ). Rotating the crystals assembly, the so-called crystal cage, about a horizontal axis, changes the energy of the exit beam.

There are two main issues with the use of a DCM for spectroscopic experiments. First, the relative alignment of the two crystals must be maintained dynamically and precisely by small adjustments to maintain perfect alignment and therefore providing optimal output flux. Precise actuators and mechanics are required to keep the two crystals aligned within the small acceptance angle for diffraction; this is usually achieved by adjusting the position of the second (down-stream) crystal. Such adjustments will inevitably cause vertical excursions of the DCM exit beam superimposed on changes due to source movements or motions induced by other optical components

Second, further changes of the exit beam height are induced when the X-ray energy is scanned. Although so called fixed-exit designs have been developed (Mills et al., 1986; Ferrer et al., 1998) that reduce vertical beam movements compared with the older pseudo channel-cut designs, beam movements of several tens of micrometres are still present over typical energy ranges needed to complete extended x-ray absorption fine structure (EXAFS) spectroscopy measurements. The effect of this change in beam height is most severe for lower $\mathrm{x}$-ray energies (i.e. below $10 \mathrm{keV}$ ) and is typically larger than the vertical beam size (300 microns). If left uncorrected the beam would be sampling different parts of the sample as a function of the energy setting. Obviously for spatial mapping of samples (Borfecchia et al., 2012) or measurements on slightly inhomogeneous samples (Mino et al., 2011) or during time resolved experiments where 
slight temperature/chemical state gradients might exist (Grunwaldt \& Schroer, 2010; Bordiga et al., 2013), this is highly undesirable.

In this paper we describe the results of experiments in which we stabilize both beam height and monochromatic beam intensity from a DCM using an extremum seeking active feedback control system. This approach is attractive because it does away with normalisation procedures that correct for changes in the input intensity as produced by the exponentially decaying synchrotron ring current or of the energy dependent output flux of the DCM itself in order to stay at the peak intensity of the DCM output. To demonstrate the effectiveness of our approach we have opted to conduct spectroscopy at the lowest practical x-ray energy at which an average hard X-ray spectroscopy beam line generally can operate. At this low energy (5 keV: Ti K-edge) a $1000 \mathrm{eV}$ energy scan requires up to about $4^{\circ}$ rotation for a $\mathrm{Si}(111)$ monochromator. The corresponding vertical swing of the exit beam is significant and large compared to the size of the beam at the sample. Energy dependent absorption and changes in monochromator energy resolution across the low energy range are more sensitive compared to experiments at higher $\mathrm{x}$-ray energies. We report the results of EXAFS measurements carried out on a Titanium containing sample with and without the two types of beam stabilisation (intensity stabilisation using extremum seeking active feedback control and beam position stabilisation using proportional feedback control) and we discuss the performance of the proposed method. The sample investigated is the precursor of a Ziegler-Natta catalyst, employed for industrially relevant olefin polymerisation processes (Seenivasan et al., 2011, 2013). Ti K-edge EXAFS measurements on this sample were particularly critical for two main reasons: i) its inhomogeneous nature and ii) the low Ti loading $(2 \mathrm{wt} \%)$.

The intensity stabilisation method differs from a MOSTAB device (Krolzig et al., 1984) that deliberately aligns the two monochromator crystals to give only about $70 \%$ of the achievable intensity. A distinct disadvantage of this approach is that a correction has to be made which depends on the synchrotron ring current and the energy setting of the DCM.

\section{Experimental setup}

The experiments were conducted at the BM26A DUBBLE group beam line at the European Synchrotron Radiation Facility (Borsboom et al., 1998; Nikitenko et al., 2008). The bending 
magnet (critical energy $E_{c}=9.6 \mathrm{keV}$ ) beam line consists of three optical components, slits to define the beam, an in situ beam position and imaging monitor (van Silfhout et al., 2011), a sample stage with two ion chambers and a 9-element fluorescence detector. An overview of the beam line used in our experiments is given in Fig. 1.

The diverging beam is collimated in the vertical direction by bending the first mirror into a cylinder that approximates the ideal parabolic shape with the source at its centre. Hence, the collimated polychromatic or 'white' x-ray beam incident on the first (upstream) crystal of the DCM has a reduced vertical angular divergence that is essentially determined by the vertical size of the source and mirror slope errors. The collimating mirror has a fixed tilt angle of $2 \mathrm{mrad}$ allowing it to capture vertically $2.4 \mathrm{~mm}$ of the radiation fan when fully using its length of $1200 \mathrm{~mm}$ whilst higher harmonic rejection is achieved by choosing one of the two optical strips on the surface (bare Si and Pt coating). These strips can be brought into the direct beam by a lateral translation. For beam energies below $15 \mathrm{keV}$ one selects the $\mathrm{Si}$ surface, above $15 \mathrm{keV}$ the Pt strip is used to achieve higher energy spectral cut-off. At its approximate distance of $24 \mathrm{~m}$ from the source, the mirror captures the full opening angle of the bending magnet radiation. The horizontal extent of the beam is defined by slits just upstream of the DCM.

The DCM crystal arrangement is that of a pseudo channel-cut crystal variety in which the horizontal rotation axis is positioned at the surface of the first crystal. The relative alignment of the second crystal with respect to the first (upstream) crystal is changed by a piezo actuator (PI, $\mathrm{P}$-140) that creates tiny rotations ( $\quad$ ) of the second crystal about a horizontal axis positioned at its surface.

Fig. 2 shows the calculated acceptance of the $\mathrm{Si}(111)$ crystals as a function of photon energy and compares it with the vertical divergence of the beam as produced by the bending magnet source. The acceptance angle was taken as the Darwin reflection curve width for the (111) and (311) Bragg reflections taking absorption into account (Als-Nielsen \& McMorrow, 2011). Bending magnet divergence was calculated using as the opening angle of the radiation cone as produced by the 'soft field'-bending magnet at BM26A installed at the ESRF. From the figure it is clear that the collimating mirror fulfils a role in allowing the full beam to 'fit' into the acceptance angle of the monochromator. We have also indicated the limiting angular divergence after collimation of the beam due to the finite vertical size of the source and mirror slope errors of approx. $4 \mu \mathrm{rad}$, 
which would be important for monochromators using the recently added $\mathrm{Si}(311)$ crystal planes at energies above approx. $25 \mathrm{keV}$.

In Fig. 3 we show the theoretical energy resolution of the BM26A beam line based on the combined resolution of DCM crystals, source type and size and collimating mirror. For the DCM crystals the energy resolution is given rom the expression for the differential form of Bragg's equation: $\quad /=/ \tan$. In our calculations of the energy resolution we have used Gaussian approximations for both light source and crystal reflection curve.

The vertical beam displacement $D$ between the white beam and the exiting monochromatic beam is a function of the Bragg angle $\theta_{B}$ and the gap $h$ (on BM26A $h=10 \mathrm{~mm}$ ) between the two crystals:

$$
D=2 h \cos \theta_{B} .
$$

For low energies (e.g. large Bragg angles), the changes in $D$ during an EXAFS scan (about a $1 \mathrm{keV}$ wide range) are considerable. For example, with a commonly used DCM gap of $10 \mathrm{~mm}$, a scan around the K-edge of Ti results in a change of vertical beam displacement of $0.5 \mathrm{~mm}$.

Downstream from the DCM is a flat silicon mirror, which can be bent to focus the monochromatic beam at the (variable) sample position with a vertical size in the focal plane of approximately $300 \mu \mathrm{m}$. Similarly to the collimating mirror, this focusing mirror has two $30 \mathrm{~mm}$ wide strips along its length, one coated with a thin layer of Platinum and the other bare Silicon. The horizontal extent of the beam at the sample is set by slits. The angle of incidence is fixed at approx. 2 mrad. The vertical position of the beam exiting the vertically focusing mirror is measured by a beam position and beam shape measurement instrument that is able to record key beam parameters during an experiment (Kachatkou et al., 2013). In brief, this instrument records the scattered radiation from a thin Kapton ${ }^{\mathrm{TM}}$ foil that is placed obliquely with respect to the incident beam. By collecting the scattered x-rays through an aperture an (enlarged) image of the footprint of the beam is recorded using an x-ray imaging sensor. For BM26A, we used a slit as an aperture because we were only interested in the vertical position of the beam (the beam line does not have a horizontal focusing capability and the beam width is set by slits just upstream of the sample). The resolution of the setup is determined by several parameters (aperture size, beam intensity and sensor noise) and is approx. $1 \mu \mathrm{m}$. 
Beam intensity was recorded just upstream of the sample position with the use of an ion chamber (FMB Oxford IC Spec DIS-0100, $I_{0}$ in Fig. 1) which was filled with a gas mixture of 5\% N2 and $95 \% \mathrm{He}$ at a pressure of 1.2 bar. The output current of the ion chamber was amplified with a trans-impedance amplifier and fed into the EXAFS control system as a pulse rate using a Voltage to Frequency converter.

\section{Feedback control}

From the previous section it is clear that active control is needed to compensate for vertical beam movement as induced by scans of the x-ray energy. In principle, one can calculate the beam shift as a function of energy and correct accordingly. This method would work if not for the fact that there are additional sources for beam shift that make it imperative to apply corrections using a feedback control loop. One common cause of additional beam shifts during energy scans are mechanical issues to keep the two DCM crystals aligned with respect to each other in terms of pitch angle. This alignment error has a significant effect on the monochromatic beam intensity. For keeping the feedback control loop, that stabilises the beam position, operating it is, therefore, imperative to control the relative alignment of the DCM crystals. By creating a fast feedback control loop that maintains DCM crystal alignment, we create a situation in which the second, slower, position control feedback loop always operates with a beam of sufficient intensity. There are several ways to correct for vertical beam shifts irrespective whether they are due to DCM energy or vertical source position changes. Adjusting the gap $h$ between the crystals such that the right hand side of equation (1) remains constant is an obvious way. Unfortunately the BM26A DCM gap was fixed at $10 \mathrm{~mm}$. We therefore opted to adjust the height of the downstream mirror without changing its tilt angle. Adjusting the vertical sample position would also be an option but this approach requires also a simultaneous motion of the vertical exit slits and is critical in cases of complex experimental set-ups such as cryobeam (Gianolio et al., 2010) or heat gun on the samples (Günter et al., 2001). Adjustments of the focusing mirror tilt angle are also possible but these cause changes in the cut-off energy of the mirror and necessitate extra changes in exit slit settings by amounts that depend on the angular changes.

There are two different methods of applying feedback control for keeping both crystals of the DCM aligned with respect to each other. The first method introduces a slight misalignment between the two crystals such that the exiting monochromatic beam is approx. $70 \%$ below the 
peak value (Krolzig et al., 1984). The DCM beam intensity as a function of the relative alignment of the two crystals - the so called rocking curve - has a width that decreases with increasing output $\mathrm{x}$-ray beam energy. Therefore, the method of aligning both crystals away from the peak reduces the higher harmonic content significantly (Als-Nielsen \& McMorrow, 2011). At either side of the rocking curve it is relatively simple to create an appropriate feedback control signal that indicates in which way and by what amount to move the two monochromator crystals with respect to each other in the case of a small deviation from the preferred position. However, this method has a distinct disadvantage: the output flux varies as a function of synchrotron ring current and as a function of the energy setting (Bragg angle). In order for this approach to be effective, the feedback control signals require elaborate normalisation procedures to keep the exact position on the rocking curve. One also sacrifices $30 \%$ of the available flux and therefore requires extra beam time to obtain the required experimental statistics.

The second method for intensity stabilisation is an extremum seeking control strategy. A paper by Krstić (Krstić \& Wang, 2000) provides the theory behind the stability of such a control scheme. Van Mellaert and Schwuttke (van Mellaert \& Schwuttke, 1970) first introduced this approach for stabilising x-ray beam intensity. More recent implementations developed specifically for DCM feedback control can be found at several synchrotron radiation facilities (Mills \& Pollock, 1980; Kudo et al., 2007).

Below we describe in detail the underlying principles of both fast extremum seeking control and the slower position control method.

\subsection{Intensity control - extremum seeking}

We have opted for a feedback control system that controls the setting of the piezo actuator to keep both crystals aligned at the top - extremum - of the rocking curve. The condition to stay at the peak of the rocking curve is not trivial to implement for several reasons. First, the extremum changes with time and selected beam energy. Secondly, if there is any slight misalignment, it will not be obvious in which direction the correction (e.g. the change in the setting of the piezo actuator) should be undertaken. There are many reasons why perfect alignment can't be maintained, particularly when the DCM energy setting is changed. For example, a change to larger Bragg angles will increase the power density of the incident beam onto the first (upstream) crystal causing a change in the thermal distortion. 
The extremum seeking control method applies a sinusoidal excitation voltage with small amplitude to the control signal to the actuator see Fig. 4. For a well-aligned system this dither signal will produce a ripple with negligible amplitude superimposed on the DCM output intensity.

The use of a periodic dither signal with small amplitude superimposed on a DC bias moves the second crystal smoothly around the set position. In the case of a misalignment, a distinctive oscillation of the DCM output intensity (ion chamber reading) will be evident. The phase comparison with the dither signal will indicate on which side of the rocking curve we are located. Our phase sensitive control scheme is shown schematically in Fig. 4. The measured beam intensity signal is amplified but only the time dependent (AC) signal is passed to a narrow band filter tuned to the frequency of the dither signal. The amplitude of this AC signal is proportional to $A R(\theta)$ where $R(\theta)$ is the derivative of the rocking curve with respect to $\theta$ and $A$ the amplitude of the dither signal. The resulting filtered signal is then compared with that of the dither signal to give a " +1 " or " -1 " depending whether there is a positive or negative phase shift between them. The ion chamber signal was read directly at the output of the trans-impedance amplifier. The signed amplitude is used as an input signal to a proportional controller whose output is summed to that of a manual bias and the dither signal before passed to the piezo actuator amplifier. Signal processing is conducted in the digital domain after the (amplified) signals have been converted using a 16-bit analogue to digital converter. The procedure for extracting the phase difference uses fast Fourier transformations (FFT) on the two measured signals. The two phases are obtained from the FFT bins belonging to that of the dominant (dither) frequency.

Our phase sensitive detection (PSD) approach differs from the traditional phase locked loop method (Mills \& Pollock, 1980; Kudo et al., 2007) in that the processing chain doesn't rely on using the DC component of the multiplication of the sinusoidal dither signal and the AC component of the measured signal. It can be shown that for the phase locked loop (PLL) method the DC component is proportional to $\frac{A^{2}}{2} R(\theta)$ (Krstić \& Wang, 2000). Particular for spectroscopic methods (such as EXAFS) it is important to keep the amplitude $A$ of the dither signal small so that the energy changes of the $\mathrm{x}$-ray beam are kept as small as possible. In this 
case, our approach provides higher sensitivity to amplitude variations than the traditional PLL method.

An approach in which the tilt of the second crystal is used to stabilise both position and intensity is a compromise that results in varying intensity levels or vertical beam shifts (Kudo et al., 2007).

\subsection{Beam position control}

For beam position control, a transmissive beam diagnostics instrument that measures beam position and beam profiles with high resolution without interfering with delivery of the beam at the sample is used. To improve signal to noise ratio of the measured signal, several individual beam profiles are added to give a time averaged reading, which is then used to give a measurement of vertical beam position by fitting the averaged profile with a Gaussian function. This procedure has been described in earlier publications (Kyele et al., 2005; van Silfhout et al., 2011; Kachatkou \& van Silfhout, 2013).

Any changes in measured beam height are corrected by adjusting the focusing mirror height actuators. Using a simple feedback control loop, corrections are made to the focusing mirror height in order to keep the beam pointing at the same spot within $2 \mu \mathrm{m}$ accuracy. Height corrections smaller than $2 \mu \mathrm{m}$ are not made in order not to pass on motor changes that are similar to their resolution.

Due to the relatively large mass the mirror correction loop was updated at relatively low frequency of approx. $0.2 \mathrm{~Hz}$. Readings of vertical position were averaged to give a measurement at the above rate and these are used to calculate mirror height corrections using a software-based proportional controller which is completely separate from the intensity feedback loop shown in Fig. 4.

\section{Results}

At a nominal energy setting of $5.8 \mathrm{keV}$, a rocking curve measurement was performed by moving the piezo actuator such that the downstream monochromator crystal was rotated about its optimal alignment with the first crystal over a range of approximately $120 \mu \mathrm{rad}$. A voltage swing of the piezo amplifier control input by $1 \mathrm{~V}$ creates a rotation $(\delta \vartheta)$ of $53 \mu \mathrm{rad}$ of the downstream monochromator crystal. The measured response is shown as open circles in Fig. 5. The thin solid 
line shows the expected shape of the curve based on theory (a convolution of two $\operatorname{Si}(111)$ reflections - Darwin curves - calculated using dynamical diffraction theory) which were scaled to match the measured amplitude. Absorption corrections were included in the calculations. The dotted line shows a Gaussian best fit to the data for comparison.

To investigate the behaviour of the PSD system, we scanned the piezo bias voltage whilst recording the amplitude of the time varying (AC) part of the beam intensity monitor signal. The oscillation amplitude superimposed on the measured output beam intensity (Fig. 5) is a direct indication of the misalignment, provided this misalignment does not extend beyond the half width of the rocking curve. The bias voltage is adjusted so that the setting of the actuator creates a perfect alignment between the two crystals.

The frequency of the dithering signal was chosen to be $6 \mathrm{~Hz}$, which is fast compared to the measurement steps during the EXAFS experiments but not too fast for the piezo amplifier that has to drive a large capacitive load. A PSD system shown in Fig. 4 was used to control the DC bias of the piezo control signal. When multiplied by the sign of the phase difference between excitation oscillations and measured beam intensity a signal is obtained that is equal to the differential of the rocking curve. The result of these measurements is shown in Fig. 5 for three amplitudes of the dither signal $(25,35$ and $50 \mathrm{mV})$. It is clear that the measured signal behaves as expected with the measured PSD signal linear proportional to the excitation amplitude.

This signal has the right characteristics to act as the input for a proportional controller because it has the right behaviour. Firstly, it indicates correctly on which side of the peak any misalignment is occurring providing a way back to the peak value. Secondly, the signal is linear proportional to the misalignment for angles of misalignment that can be as large as approx. half of the width of the full rocking curve. Finally, the signal passes through zero for optimal alignment.

Performing a sinusoidal oscillation of the downstream DCM crystal causes periodic changes in the energy of the photon beam. It is important to understand the effects of the intensity stabilisation method on the energy resolution and how this affects the measured EXAFS data. Typical EXAFS scans use a constant step size $(\Delta k)$ in the photo-electron wave vector of $0.05 \AA^{-}$ 1. This step size is small enough to sample real space distances beyond $20 \AA$ well beyond the usually required distance. Using the expression for the conversion between photo-electron wave vector $k$ and the x-ray energy ( $k=0.51 \sqrt{E-E_{0}}$ with $k$ in $\AA^{-1}$ and $E, E_{0}$ in eV), one readily finds 
that for energies, $E$, of $30 \mathrm{eV}$ above the edge the equivalent energy change is similar to the induced energy variation due to the monochromator dithering with an amplitude such as used during the experiments. With the monochromator at the Ti K-edge energy $\left(\mathrm{E}_{0}=5 \mathrm{keV}\right)$ the dithering signal induces energy changes of $0.88,1.23$ and $1.77 \mathrm{eV}$ for amplitudes of 25,35 and $50 \mathrm{mV}$ respectively. For higher x-ray energies the $0.05 \AA^{-1}$ step in wave vector quickly grows towards $5.5 \mathrm{eV}$ at $5.8 \mathrm{keV}$. For reference, the inherent energy resolution of the beam line used is $0.7 \mathrm{eV}$ at $5 \mathrm{keV}$.

With the intensity feedback loop running at a $2 \mathrm{~Hz}$ update rate we recorded the ion chamber reading as a function of energy while a Titanium K-edge EXAFS scan was performed. For comparison we also did the same experiment with the feedback switched off. Before this latter measurement, the two crystals were manually aligned at a single energy of approximately $5.2 \mathrm{keV}$, representing the middle of a typical Ti K-edge EXAFS scan (4.7-5.7 keV). Fig. 6a shows the result of the two experiments and compares them with the expected (scaled) output of the beam line. The enhanced performance of the intensity feedback control loop is evidenced by the provision of a higher beam flux throughout the whole EXAFS experiment. This will improve the signal-to-noise ratio in the spectroscopy data significantly, particularly in the case of spectra collected in fluorescence mode on diluted samples. What is surprising, however, is the large deviation between theory and measured flux at lower energies. Particularly at energies below the Ti-edge a significant drop-off is seen in the ion chamber reading. In the calculations for the expected beam intensity using the ESRF's SRW software package, we have accounted for the absorption of the Beryllium windows that were taken to be free from impurities and deposits (such as carbon). At these low beam energies careful consideration to impurity and air absorption is required to predict the measured intensity. The same behaviour was apparent when we measured the rocking curves for five energies across the energy range (see Fig. 6b). Any impurities in the beam line vacuum windows, reflecting surfaces (of mirrors and monochromator crystals) and non-linear behaviour of the detectors can impose experimental problems and hence, when working at these low energies, experimental hygiene is of utmost importance. It is likely that mirror contamination is the most likely cause for the reduced measured flux at the lower $\mathrm{x}$ ray energies; this beamline has been in operation for about a decade with high levels of user operation. 
Measurements of vertical beam position during EXAFS scans with (and without) position feedback enabled were published elsewhere (Kachatkou et al., 2013) and show that a positional stability of $4.1 \mu \mathrm{m}$ (root mean square) is achieved during the scan which corresponds to the threshold limit $( \pm 2 \mu \mathrm{m}$ ) set in software to prevent sending corrections to the actuator system that are beyond its positional precision.

EXAFS measurements were performed on an in-homogeneous sample having a $2 \mathrm{wt} \%$ Tiloading in fluorescence mode using a 9-element monolithic Ge detector. Four different scans were taken under four different feedback scenarios: no feedback, optimised monochromatic intensity $\left(I_{0}\right)$, feedback to keep beam height constant and feedback on intensity and position. For the optimised intensity feedback runs, a $35 \mathrm{mV}$ amplitude of the dither signal was used which corresponds to a $1.23 \mathrm{eV}$ change in energy. The raw EXAFS data, shown in Fig. 7a, are characterised by a different slope of absorption coefficient $\mu$ as a function of energy. The difference in the EXAFS oscillations is more evident by looking to the extracted EXAFS signals, $k^{3} \chi(k)$, as a function of $k$ (Fig. 7b). Below $k=6.5 \AA^{-1}$ the four data collections methods generate almost equivalent data, differing only by some $10 \%$ in the amplitude, but exhibiting the same phase. Starting from $6.5 \AA^{-1}$ the $k^{3} \chi(k)$ functions start to be distorted both in phase and in amplitude. The important beat around $k=8.5 \AA^{-1}$ is significantly suppressed when the two feedbacks are not simultaneously operative. The error in the phase of the $k^{3} \chi(k)$ functions in the 9.0 - $10.5 \AA^{-1}$ is also remarkable. Obviously, problems related to a non-optimal data collection are more evident in the high $k$-region, where the EXAFS signal is less intense. The Fourier Transformed $k^{3} \chi(k)$ functions (calculated in the $\Delta k=2.0-10.0 \AA^{-1}$ range) are clearly different for spectra collected with different feedback configuration, in both modulus (Fig. 7c) and imaginary parts of (Fig. 7d). It is thus clear that a quantitative EXAFS data analysis on the investigated sample will be affected by severe systematic errors for the data obtained without using the feedback and positional control.

\section{Discussion}

The performance of both feedback loops was satisfactory keeping both beam intensity and position optimal during the full EXAFS scans even though major corrections in terms of monochromator alignment and beam height had to be made. Over the range between 4.7 and $5.8 \mathrm{keV}$ the beam height was corrected by an amount of $0.66 \mathrm{~mm}$, close to the calculated amount 
based on a crystal gap of $10 \mathrm{~mm}$. Corrections to the piezo setting amounted to half the width of the rocking curve at $5 \mathrm{keV}$.

For near edge (XANES) scans, the highest energy resolution is required and dithering of the monochromator crystals should be avoided. The reduced scan range for XANES results in minimal intensity changes as evidenced by our measurements shown in Fig 6a. The use of the dithering of the monochromator crystal is therefore not needed allowing for the highest energy resolution for near edge spectroscopy scans.

Judging from the sharp pre-edge peak, our method does not induce a noticeable energy shift due to asymmetrical movements or drifts of the monochromator mechanism. Comparing the pre-edge peak width for the runs with and without feedback we do not see a significant broadening of the peak due to a reduced energy resolution with the regular steps in energy change of the incident beam.

The periodic dither signal causes periodic changes in both energy and vertical position of the beam, which are taking place at a much higher rate than the typical stepped EXAFS data collection strategy that keeps the energy setting constant for at least 1 second, much more for fluorescence collections on diluted samples. The feedback loops are implemented in software, which gives the system a high level of flexibility allowing quick changes to dither frequency and amplitude whilst providing ways to synchronise any actuator changes with the EXAFS measurement protocol and timing.

In conclusion, we have presented a dual feedback approach, which has shown to improve beam delivery particularly important for EXAFS scans at low energies such as the Ti K-edge. This development is of particular importance to users that are investigating inhomogeneous samples or those that want to create spatially resolved measurements of samples. The proposed feedback system should be of interest to multiple beam lines not just those that are designed for spectroscopy. The phase sensitive feedback scheme presented is relatively simple and requires no expensive components such as lock-in amplifiers. Beam position changes and monochromator crystal alignment are seen across the spectrum of x-ray beam lines.

Our work offers uncompromised beam intensity delivered at the same sample location irrespective of beam energy or source motion. 
Acknowledgements We thank NWO for providing beam time and travel funds. BM26A staff, especially Dipanjan Banerjee and Dirk Detollenaere, is acknowledged for their support.

References

Bordiga, S., Groppo, E., Agostini, G., van Bokhoven, J. A., \& Lamberti, C. (2013). Chem. Rev. 113, 1736-1850.

Borfecchia, E., Mino, L., Gianolio, D., Groppo, C., Malaspina, N., Martinez-Criado, G., Sans, J. A., Poli, S., Castelli, D., \& Lamberti, C. (2012). J. Anal. At. Spectrom. 27, 1725-1733.

Borsboom, M., Bras, W., Cerjak, I., Detollenaere, D., Glastra van Loon, D., Goedtkindt, P., Konijnenburg, M., Lassing, P., Levine, Y. K., Munneke, B., et al. (1998). J. Synchrotron Radiat. 5, 518520.

Ferrer, J. L., Simon, J. P., Bérar, J. F., Caillot, B., Fanchon, E., Kaïkati, O., Arnaud, S., Guidotti, M., Pirocchi, M., \& Roth, M. (1998). J. Synchrotron Radiat. 5, 1346-1356.

Gianolio, D., Groppo, E., Vitillo, J. G., Damin, A., Bordiga, S., Zecchina, A., \& Lamberti, C. (2010). Chem. Commun. 46, 976-978.

Grunwaldt, J.-D. \& Schroer, C. G. (2010). Chem. Soc. Rev. 39, 4741-4753.

Günter, M. M., Ressler, T., Jentoft, R. E., \& Bems, B. (2001). J. Catal. 203, 133-149.

Kachatkou, A., Kyele, N., Scott, P., \& Silfhout, R. van (2013). J. Phys. Conf. Ser. 425, 042002.

Kachatkou, A. \& van Silfhout, R. (2013). Opt. Express. 21, 4291-4302.

Krolzig, A., Materlik, G., Swars, M., \& Zegenhagen, J. (1984). Nucl. Instruments Methods Phys. Res. 219, 430-434.

Krstić, M. \& Wang, H.-H. (2000). Automatica. 36, 595-601.

Kudo, T., Tanida, H., Inoue, S., Hirono, T., Furukakwa, Y., Nishino, Y., Suzuki, M., \& Ishikawa, T. (2007). Aip Conf. Proc. 879, 954-958.

Kyele, N. R., Decanniere, K., \& van Silfhout, R. G. (2005). J. Synchrotron Radiat. 12, 800-806.

Van Mellaert, L. J. \& Schwuttke, G. H. (1970). Phys. Status Solidi. 3, 687-696.

Mills, D. M., Henderson, C., \& Batterman, B. W. (1986). Nucl. Instruments Methods Phys. Res. Sect. Accel. Spectrometers Detect. Assoc. Equip. 246, 356-359.

Mills, D. \& Pollock, V. (1980). Rev. Sci. Instrum. 51, 1664 -1668.

Mino, L., Gianolio, D., Agostini, G., Piovano, A., Truccato, M., Agostino, A., Cagliero, S., Martinez-

Criado, G., d' Acapito, F., Codato, S., et al. (2011). Small. 7, 930-938. 
Als-Nielsen, J. \& McMorrow, D. (2011). Elements of Modern X-ray Physics John Wiley \& Sons.

Nikitenko, S., Beale, A. M., van der Eerden, A. M. J., Jacques, S. D. M., Leynaud, O., O’Brien, M. G.,

Detollenaere, D., Kaptein, R., Weckhuysen, B. M., \& Bras, W. (2008). J. Synchrotron Radiat. 15, 632640.

Pascarelli, S., Boscherini, F., D’Acapito, F., Hrdy, J., Meneghini, C., \& Mobilio, S. (1996). J. Synchrotron Radiat. 3, 147-155.

Seenivasan, K., Gallo, E., Piovano, A., Vitillo, J. G., Sommazzi, A., Bordiga, S., Lamberti, C., Glatzel, P., \& Groppo, E. (2013). Dalton Trans.

Seenivasan, K., Sommazzi, A., Bonino, F., Bordiga, S., \& Groppo, E. (2011). Chem. - Eur. J. 17, 86488656.

van Silfhout, R., Kachatkou, A., Kyele, N., Scott, P., Martin, T., \& Nikitenko, S. (2011). Opt. Lett. 36, 570-572.

List of figures

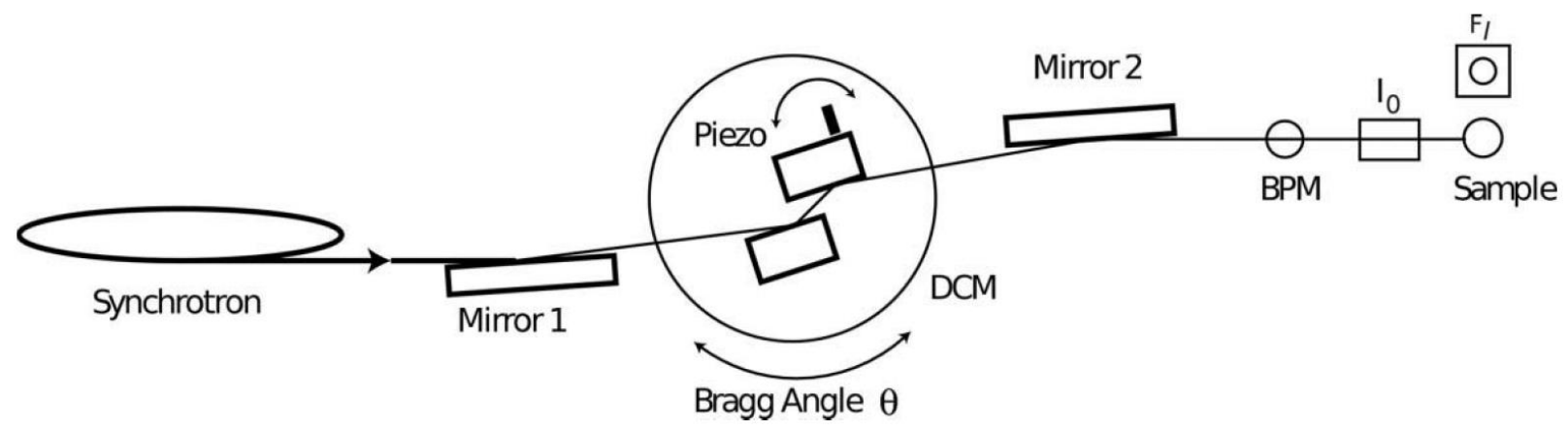

Figure 1 An overview of BM26A showing the main optical elements, beam position monitor (BPM), fluorescence detector $\left(\mathrm{F}_{1}\right)$ and ion chamber $\left(\mathrm{I}_{0}\right)$ used in the proposed feedback scheme. Slit systems are not shown. The drawing is not to scale. The second ion chamber that records the transmitted beam downstream of the sample is not shown. 


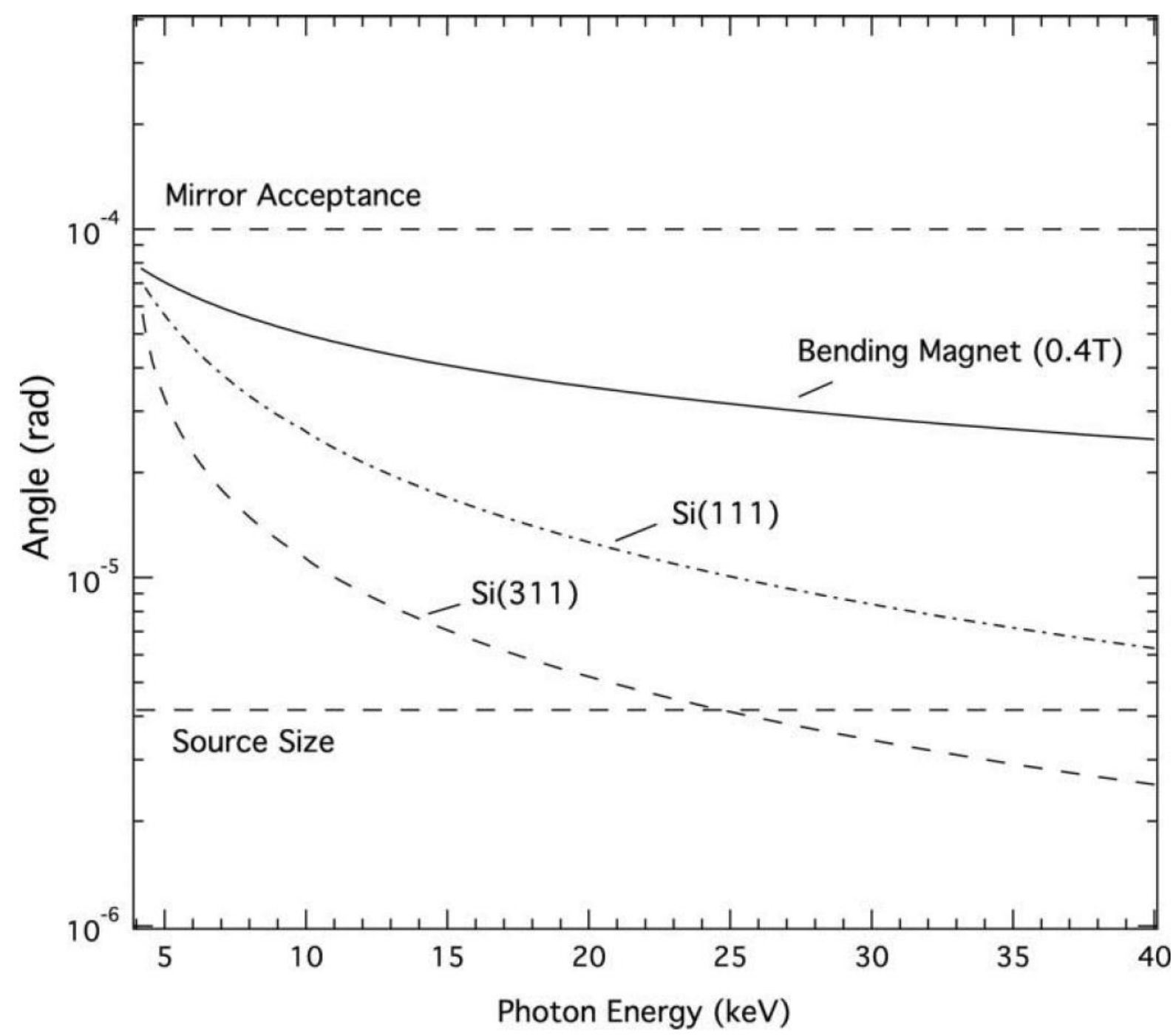

Figure 2 Calculated acceptance angle of monochromator compared to the divergence of the source. Using the silicon (111) reflection, the monochromator can not accept the full vertical fan of radiation from the source for x-ray energies above $4 \mathrm{keV}$. For reference we have also given the divergence caused by the finite vertical size of the source. 


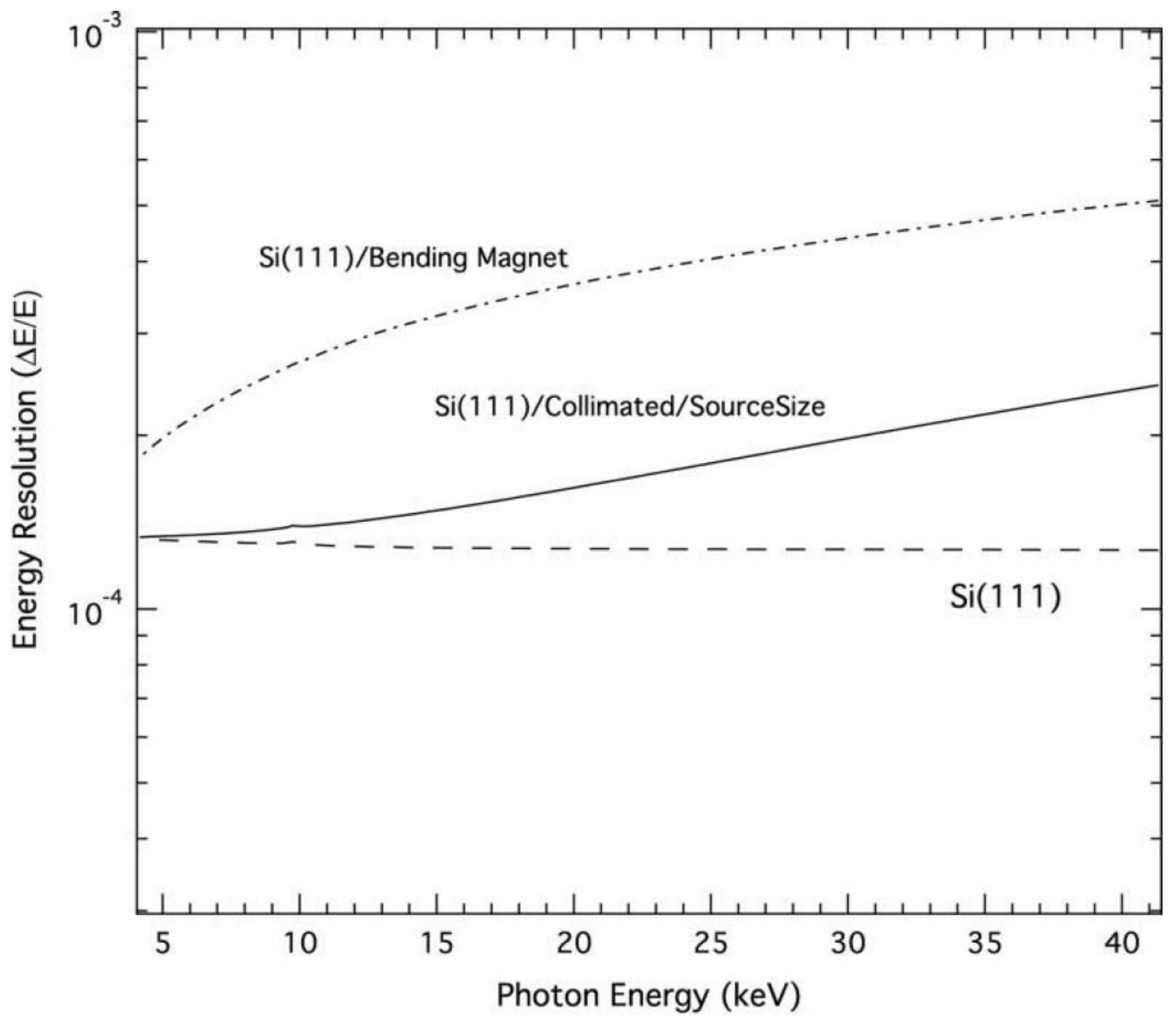

Figure 3 Energy resolution calculations for the intrinsic Si(111) crystal monochromator (dashed), the combination of monochromator and divergent bending magnet source (dash-dotted) and that for the monochromator with the collimating mirror taking into account the finite size of the source. 


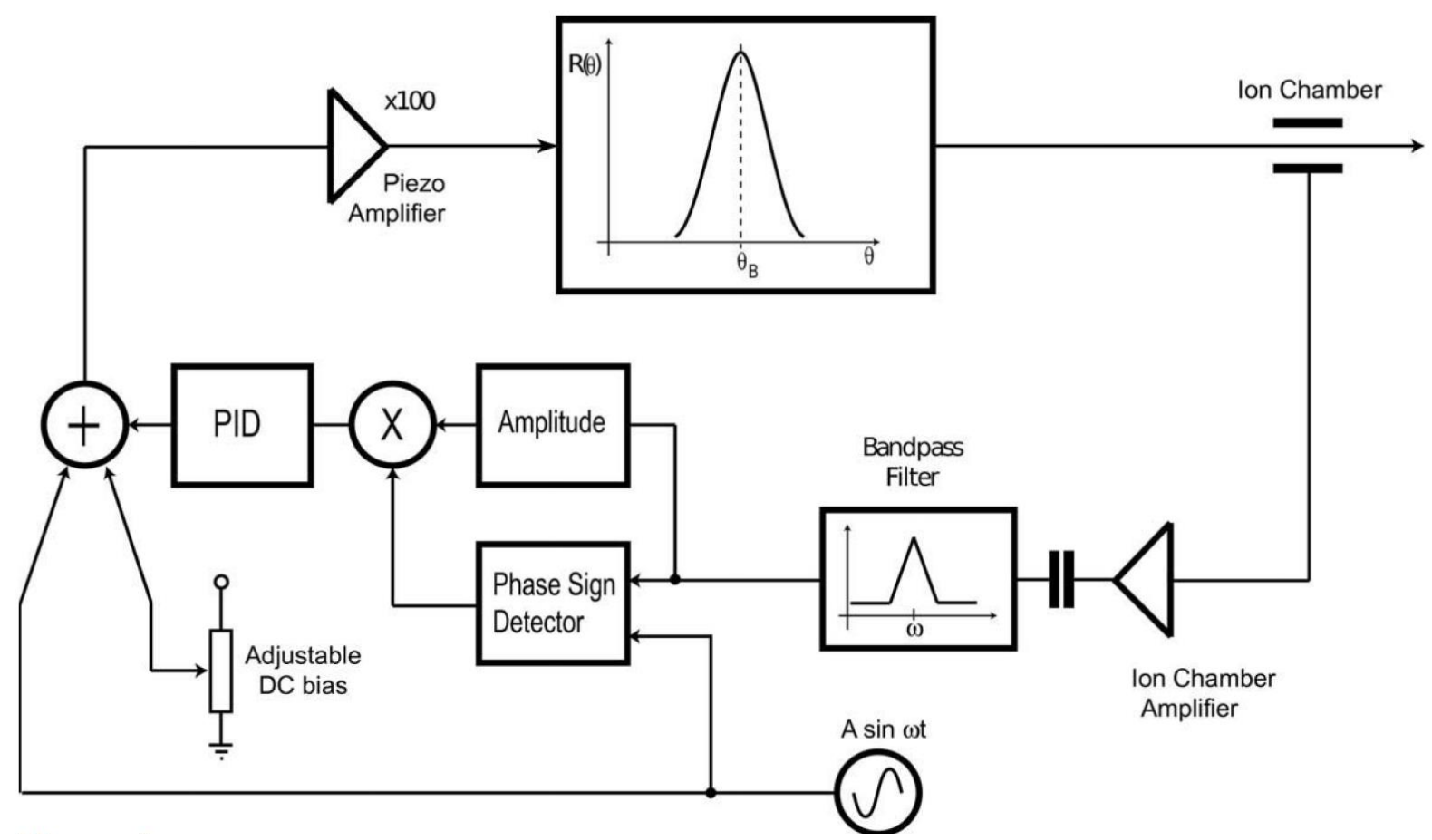

Figure 4 Diagram of the phase-sensitive detection (PSD) scheme used in the experiments. A sinusoidal varying excitation is added to a bias voltage used to set the piezo actuator. The filtered $\mathrm{I}_{0}$ signal is compared in phase with the excitation signal and its amplitude is measured. The output of a PID controller adjusts the bias voltage to keep the two monochromator crystals aligned optimally. The input of the PID controller is derived from the variation in the monochromator output as measured by an ion chamber. 


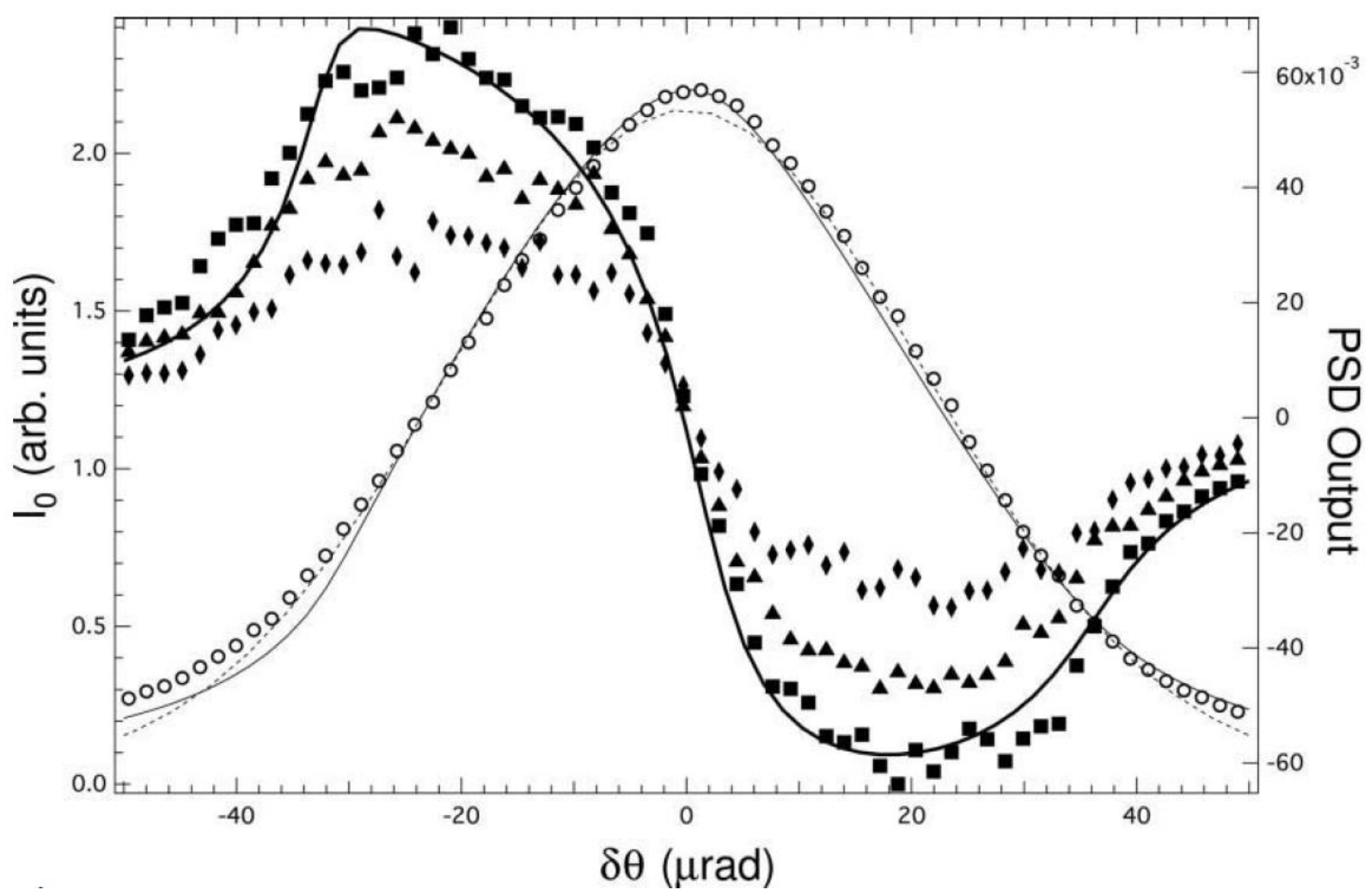

Figure 5 Measured rocking DCM curve (open circles). The filled markers denote the output of the phase sensitive detection (PSD) system using three different amplitudes of the excitation or dither signal (squares: $50 \mathrm{mV}$; triangles: $35 \mathrm{mV}$ and diamonds: $25 \mathrm{mV}$ ). The output of the PSD system is the product of the measured signal amplitude and the sign of the phase difference between the dither signal and the measured ion chamber AC signal. The thick solid line shows the differentiated theoretical rocking curve that was scaled to the result obtained with a dither signal with an amplitude of $50 \mathrm{mV}$. 


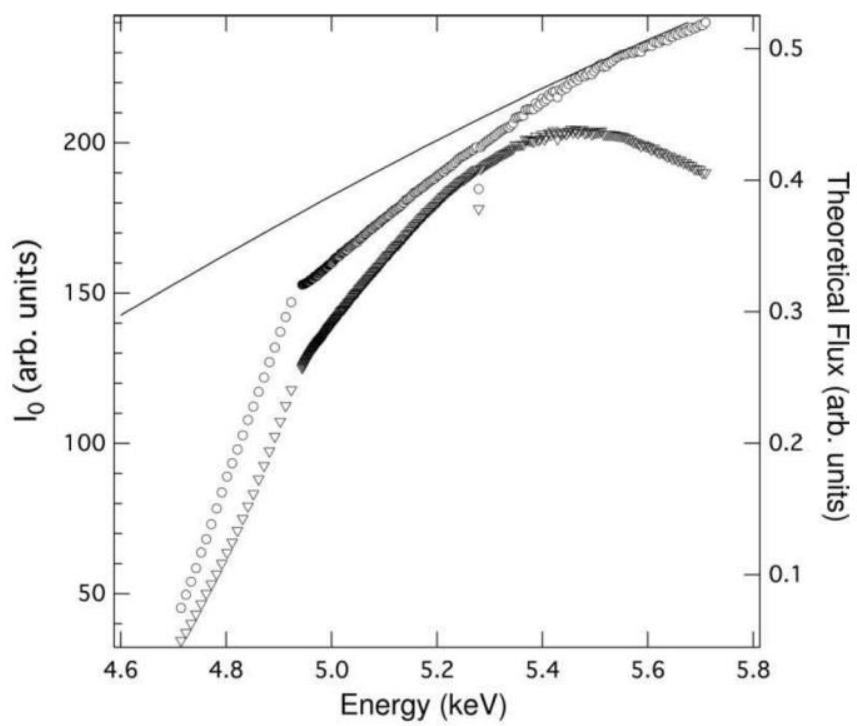

(a)

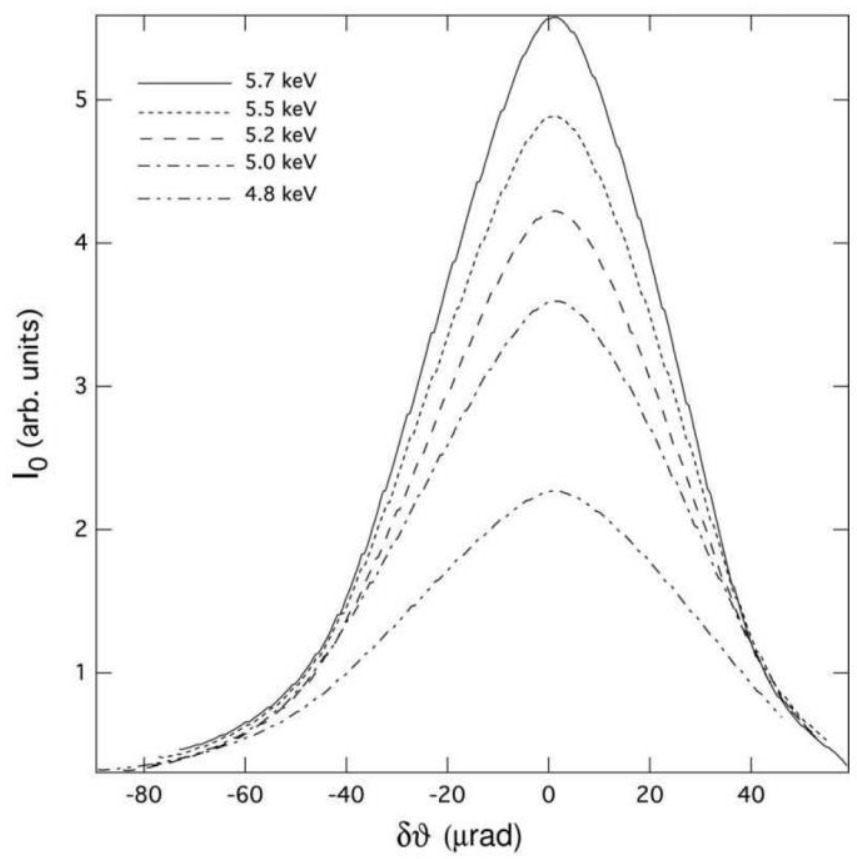

(b)

Figure 6 a) Beam intensity measurement with the ion chamber at the sample position during an EXAFS scan. The open circles denote a measurement taken with intensity and position feedback activated whereas the triangles indicate the same measurement without feedback. For the measurement without feedback the monochromator was manually aligned at a single energy (5.2 keV). Just above $5.2 \mathrm{keV}$ beam intensity is lost due to the excitation of another reflection of the silicon crystals. The solid line indicates the theoretical behaviour of the beam line. b) Rocking curves of the DCM for five energies highlighting the issue of fast degradation of produced beam intensity as a function of low energy. 

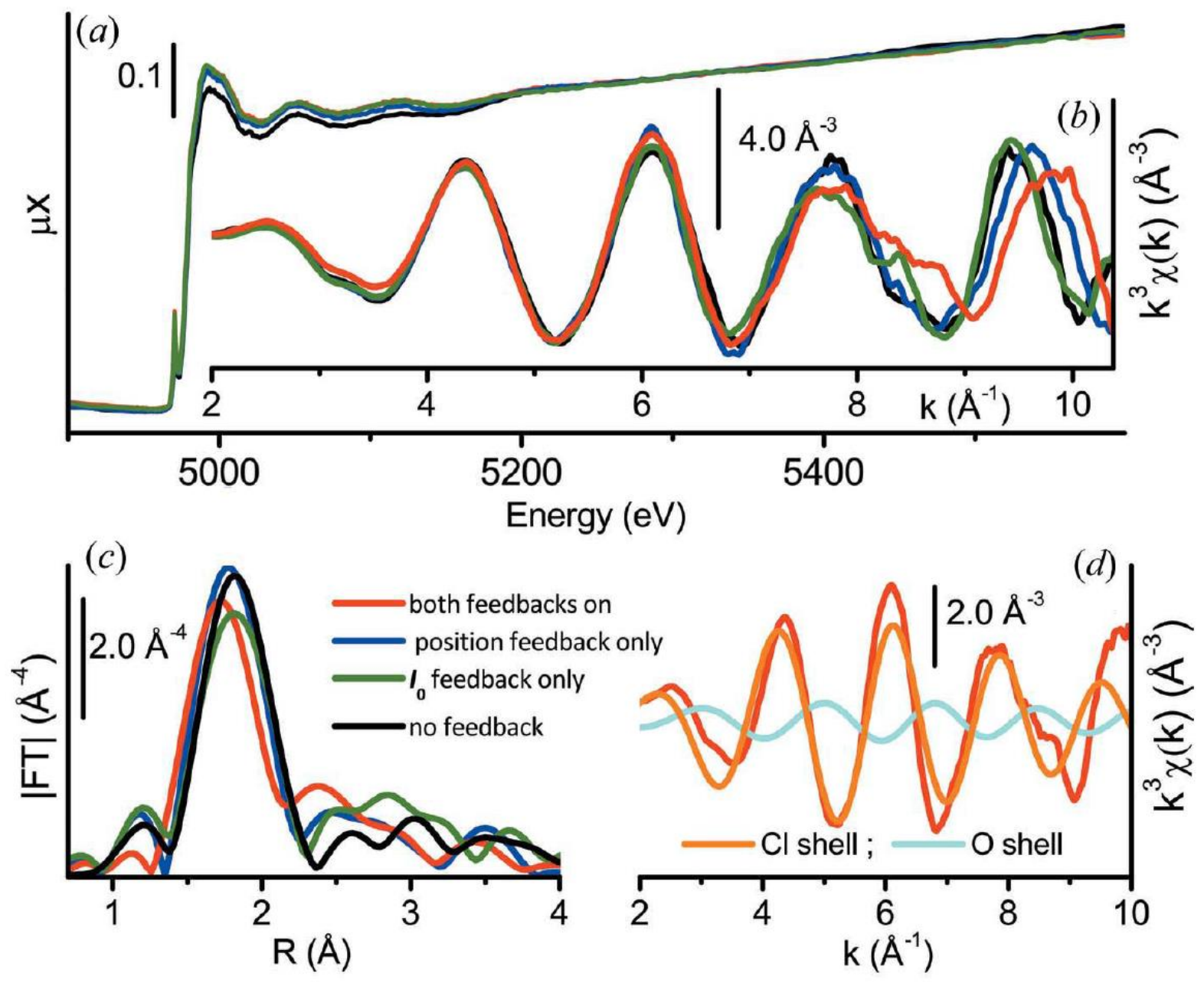

Figure 7 Rough EXAFS spectra (part a) and processed $k^{3} \chi(k)$ data (part b) of a inhomogeneous Ti containing compound measured in presence of various implementations of the feedback scheme as presented in this paper. For clarity, the $\mathrm{k}^{3} \chi(\mathrm{k})$ curves have been smoothed. The modulus and imaginary parts of the corresponding Fourier Transformed $\mathrm{k}^{3} \chi(\mathrm{k})$ spectra (in the $\Delta \mathrm{k}=2.0-10.0 \AA^{-1}$ range) are shown in parts c) and d) respectively. 